\title{
Change of the kinetics of solidification and microstructure formation induced by convection in the Ni-Al system
}

\author{
S. Reutzel ${ }^{\mathrm{a})}$ and H. Hartmann \\ Institut für Experimentalphysik IV, Ruhr-Universität, 44780 Bochum, Germany and Institut für \\ Materialphysik im Weltraum, Deutsches Zentrum für Luft- und Raumfahrt (DLR), 51170 Cologne, Germany \\ P. K. Galenko, S. Schneider, and D. M. Herlach \\ Institut für Materialphysik im Weltraum, Deutsches Zentrum für Luft- und Raumfahrt (DLR), \\ 51170 Cologne, Germany
}

(Received 2 May 2007; accepted 26 June 2007; published online 27 July 2007)

\begin{abstract}
The purpose of the present work was to measure the velocity of dendrite growth in undercooled $\mathrm{Ni}-\mathrm{Al}$ alloy melts as a function of undercooling. The experiments were performed both by containerless electromagnetic levitation on Earth and under reduced gravity conditions during parabolic flight campaigns. While under terrestrial conditions, strong magnetic fields are required to compensate the gravitational force, the forces to compensate disturbing accelerations are decreased by orders of magnitude in reduced gravity. In turn, the alternating electromagnetic fields induce convection, which is strong under terrestrial conditions while much weaker in reduced gravity. The heat and mass transport in front of the solid-liquid interface during solidification controls the dynamics of dendrite growth. By comparing results obtained on Earth and in reduced gravity, it was demonstrated that the change of transport conditions by convection significantly alters the kinetics of solidification and the evolution of grain refined microstructures at undercoolings less than 100 K. (C) 2007 American Institute of Physics. [DOI: 10.1063/1.2760154]
\end{abstract}

The dynamics of solidification controls the microstructure development of materials. It is therefore essential to have detailed understanding and knowledge of the parameters and processes of the kinetics during solidification of melts in order to improve production processes and the design of metallic materials. One may distinguish between single-phase solidification and multiple-phase formation. While the former process takes place via the growth of dendrites, the latter one is connected with more complex eutectic or peritectic reactions. During the past, detailed models became available to describe dendrite growth kinetics, and experimental techniques were developed to measure dendrite growth velocities in undercooled melts. ${ }^{1}$ It is well established that the kinetics of dendrite growth is governed by heat and mass redistribution in front of the solid/liquid interface. At large undercoolings, the atomic attachment kinetics as well plays an increasingly important role for solidification dynamics from the liquid to the solid. On the other hand, the heat and mass transport at the solidification front is influenced by convection as, e.g., by natural convection, but even more drastically by forced convection in alternating electromagnetic fields.

In the present work electromagnetic levitation was applied to containerlessly undercool and solidify melts of intermetallic $\mathrm{Ni}_{50} \mathrm{Al}_{50}$ and $\mathrm{Ni}_{60} \mathrm{Al}_{40}$ alloys. Drops in a diameter of $6 \mathrm{~mm}$ were placed in a levitation coil powered by a rf generator. The drop was freely suspended and containerlessly undercooled until its crystallization was spontaneously initiated. The advancement of the solidification front was directly observed by a high-speed camera (Photron Fastcam Ultima APX) at a frequency of up to 120000 frames/s, which allows a quantitative determination of the growth velocity. ${ }^{2}$ The temperature of the sample was measured by a two-color

\footnotetext{
${ }^{a)}$ Electronic mail: sven.reutzel@dlr.de
}

pyrometer so that the growth velocity was determined as a function of undercooling. More experimental details are given elsewhere. ${ }^{3}$ In addition, analogous experimental investigations were performed using an electromagnetic positioning device for containerless processing in reduced gravity (called TEMPUS). This facility was integrated into an aircraft (Airbus A300) which performs about 31 parabolas per day in a direct sequence, each of them providing a time of reduced gravity of about 20-22 s. The TEMPUS facility differs only in one technical detail from the electromagnetic levitation facility; i.e., two independently working coil systems were designed, one for positioning (quadrupole field) and another one for heating (dipole field). All other subsystems are comparable with the electromagnetic levitation apparatus used on Earth. More details on TEMPUS are given in the literature. ${ }^{4}$

The equiatomic alloy $\mathrm{Ni}_{50} \mathrm{Al}_{50}$ was chosen for the investigations on growth kinetics. This alloy melts congruently and forms an intermetallic B2 phase under equilibrium conditions. It can be processed properly by electromagnetic levitation. As a high temperature material with extraordinary hardness and good corrosion resistance, the intermetallic B2 phase is of particular interest. ${ }^{5}$ Intermetallics form ordered superlattice structures during solidification, which requires at least a short-range atomic diffusion at the solid-liquid interface. This leads to sluggish growth dynamics at least at small and intermediate undercoolings. ${ }^{6}$ At larger undercoolings, the driving force for rapid solidification increases, and a transition from an ordered to a disordered growth might occur. This leads to the formation of a disordered superlattice structure with an essentially improved ductility. At the same time, the temperature characteristics of the growth velocityundercooling relation change since beyond the region of an ordered growth, thermal diffusivity limits the dynamics of solidification. The thermal diffusivity is by orders of magni- 